\title{
Cardiovascular operations for Loeys-Dietz syndrome: Intermediate-term results
}

\author{
Nishant D. Patel, MD, ${ }^{\mathrm{a}}$ Todd Crawford, MD, ${ }^{\mathrm{a}}$ J. Trent Magruder, MD, ${ }^{\mathrm{a}}$ Diane E. Alejo, BA, ${ }^{\mathrm{a}}$ \\ Narutoshi Hibino, MD, James Black, MD, ${ }^{\mathrm{b}}$ Harry C. Dietz, MD, ${ }^{\mathrm{c}}$ Luca A. Vricella, MD, ${ }^{\mathrm{a}}$ and \\ Duke E. Cameron, $\mathrm{MD}^{\mathrm{a}}$
}

\begin{abstract}
Objectives: Early experience with Loeys-Dietz syndrome (LDS) suggested an aggressive aortopathy with high risk of aneurysm dissection and rupture at young ages and at smaller aortic diameters than in other connective tissue disorders. We reviewed our experience with LDS to re-examine our indications and outcomes of surgical management.

Methods: We reviewed all patients with a diagnosis of LDS who underwent cardiovascular surgery at our institution. The primary endpoint was mortality, and secondary endpoints included postoperative complications and need for reintervention.

Results: Seventy-nine operated patients with LDS were identified. Mean age at first operation was 25 years, $39(49 \%)$ were female, and $38(48 \%)$ were children (age $<18$ years). Six $(8 \%)$ patients presented with acute dissection. Five $(6 \%)$ patients had a bicuspid aortic valve, and all presented with an ascending aortic aneurysm with a mean root diameter of $3.5 \mathrm{~cm}$. Twenty $(25 \%)$ patients had a previous sternotomy. Sixty-five ( $82 \%$ ) patients underwent aortic root replacement, of whom 52 underwent a valve-sparing operation and 4 had concomitant arch replacement. Mean aortic root diameter in this group was $4.2 \mathrm{~cm}$. Nine (11\%) patients underwent aortic arch replacement, 2 (3\%) had isolated ascending aorta replacement, and $3(4 \%)$ underwent open thoracoabdominal repair. There were $2(3 \%)$ operative and 8 late deaths. Nineteen patients underwent subsequent operations for late aneurysm and/or dissection. Mean follow-up was 6 years (range 0-24 years). Kaplan-Meier survival was $88 \%$ at 10 years.
\end{abstract}

Conclusions: Growing experience with LDS has confirmed early impressions of its aggressive nature and proclivity toward aortic catastrophe. Surgical outcomes are favorable, but reintervention rates are high. Meticulous follow-up with cardiovascular surveillance imaging remain important for management, particularly as clinical LDS subtypes are characterized and more tailored treatment is developed. (J Thorac Cardiovasc Surg 2017;153:406-12)

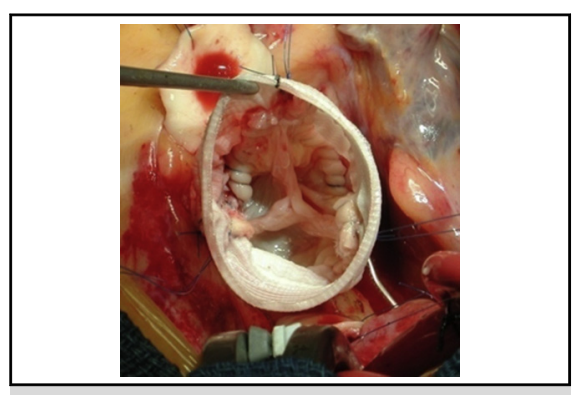

Reimplantation valve-sparing root replacement.

\section{Central Message}

Growing experience with Loeys-Dietz syndrome has confirmed the early impressions of its aggressive nature and proclivity toward aortic catastrophe. Surgical outcomes are favorable, but reintervention rates are high. Meticulous follow-up with cardiovascular surveillance imaging remain important for management, particularly as clinical subtypes are characterized and more tailored treatment is developed.

\section{Perspective}

Loeys-Dietz syndrome is an aggressive aortopathy with high risk for aneurysm rupture and dissection at younger ages and smaller aortic diameters than in other connective tissue disorders. The early and intermediate-term surgical outcomes are favorable, but meticulous longterm follow-up is critical, given the high incidence of reintervention.

See Editorial Commentary page 413.
From the ${ }^{\mathrm{a}}$ Division of Cardiac Surgery and ${ }^{\mathrm{b}}$ Division of Vascular Surgery, Department of Surgery, and ${ }^{\mathrm{c}}$ Department of Medicine, The McKusick-Nathans Institute of Genetic Medicine, The Johns Hopkins Medical Institutions, Baltimore, Md.

Read at the 96th Annual Meeting of The American Association for Thoracic Surgery, May 14-18, 2016, Baltimore, Maryland.

Received for publication May 28, 2016; revisions received Oct 2, 2016; accepted for publication Oct 5, 2016; available ahead of print Dec 9, 2016.

Address for reprints: Duke E. Cameron, MD, Division of Cardiac Surgery, Director of The Albert "Cubby" and Dana Broccoli Aortic Surgery Center, 1800 Orleans St, Zayed 7107, Baltimore, MD 21287 (E-mail: npatel4@jhmi.edu).

0022-5223/\$36.00

Copyright (c) 2016 by The American Association for Thoracic Surgery

http://dx.doi.org/10.1016/j.jtcvs.2016.10.088
Loeys-Dietz syndrome (LDS) is an autosomal-dominant connective tissue disorder first described in 2005; it is characterized by generalized arterial tortuosity, hypertelorism,

Scanning this QR code will take you to a procedural video. To view the AATS 2016 Webcast, see the URL next to the video thumbnail. 


\section{Abbreviations and Acronyms \\ LDS = Loeys-Dietz syndrome \\ SMAD3 $=$ mothers against decapentaplegic homolog 3 \\ TGFBR $=$ transforming growth factor beta receptor}

and a broad/bifid uvula or cleft palate. ${ }^{1}$ Mutations in the transforming growth factor beta receptor (TGFBR) 1 and 2, mothers against decapentaplegic homolog 3 (SMAD3), and transforming growth factor beta ligand genes are known to cause LDS, with variable expression of cardiovascular, craniofacial, cutaneous, and skeletal features. Of utmost importance is the widespread arterial involvement, tortuosity, and risk of dissection/rupture, which occur at younger ages and at smaller aortic diameters than many other connective tissue disorders.

In our initial experience with LDS, ${ }^{2}$ we reported on the first 71 patients with LDS from 2 institutions, of whom 21 had surgery. From this early experience, we discovered the malignant nature of aortic disease in LDS and recommended an aggressive surgical approach to prevent aortic catastrophe. In this report. we share our updated experience to re-examine indications and outcomes for the surgical management for LDS.

\section{METHODS}

After obtaining institutional review board approval, we retrospectively reviewed the medical records of all patients with the diagnosis of LDS who underwent cardiovascular surgery at The Johns Hopkins Hospital. A waiver for individual consent was granted by our institutional review board. The diagnosis of LDS was confirmed with genetic analysis and clinical phenotype in collaboration with colleagues from the McKusick Department of Medical Genetics and the Connective Tissue Disorders Clinic at the Johns Hopkins Hospital. Some patients in this series were evaluated and treated before the initial description of LDS and were presumed to have Marfan syndrome and were later reclassified as LDS after genetic testing.

Data were collected from hospital records, operative reports, discharge summaries, and outpatient follow-up records. Variables collected included demographics, medical and surgical history, operative data, pre- and postoperative cardiovascular imaging, postoperative complications, and further interventions on the arterial tree. Mortality data were obtained from autopsy records (when available), death certificates, and/or outpatient reports. Statistical analyses were performed with STATA 9.0 software (StataCorp LP, College Station, Tex). Continuous variables are reported as means \pm standard deviation, unless otherwise noted. Follow-up was $100 \%$ complete and mean follow-up was 5.6 years for a total of 445 patient years.

\section{RESULTS}

\section{Preoperative Clinical Characteristics}

From February 1989 to July 2015, 79 patients with a confirmed diagnosis of LDS were identified. Thirty-nine $(49.4 \%)$ were female. Mean age at first operation at our institution was $24.9 \pm 17.5$ years. Thirty-eight $(48.1 \%)$ were children younger than 18 years of age. Twenty $(25.3 \%)$ had a previous sternotomy. Previous operations included 1 ascending repair, 1 repair of type A dissection, 1 ascending replacement/aortic valve replacement, 1 coronary artery bypass grafting/aortic valve replacement, 1 atrial septal defect closure, 5 aortic root replacements, 5 valve-sparing root replacements, 1 aortic valve repair, and 4 mitral valve repairs. All previous operations were performed at outside institutions. Six $(7.6 \%)$ patients presented with a type A aortic dissection.

\section{Operative Data}

Index operations in this cohort of 79 patients with LDS included $9(11.4 \%)$ arch replacements, $2(2.5 \%)$ ascending aorta replacements, $3(3.8 \%)$ open thoracoabdominal repairs, and $65(82.3 \%)$ aortic root replacements. Of the 65 patients who had aortic root replacement, $52(80.0 \%$; $\mathrm{n}=65)$ had valve-sparing root replacement and 13 $(20.0 \%)$ had composite valve-graft root replacement. Mean preoperative aortic root diameter for these 65 patients was $4.17 \pm 0.97 \mathrm{~cm}$. Among the 52 patients with LDS who had valve-sparing root replacement, $45(86.5 \%)$ had reimplantation with a Valsalva graft (Figure 1), $2(3.8 \%)$ had reimplantation with a straight tube graft, $2(3.8 \%)$ had remodeling, and $3(5.8 \%)$ had a Florida sleeve procedure.

Concomitant procedures for the 65 patients that had aortic root replacement include closure of a patent foramen ovale in $23(35.4 \%)$, closure of a patent ductus arteriosus in $3(4.6 \%)$, aortic arch repair in $4(6.2 \%)$, aortic valve repair in $3(4.6 \%)$, and mitral valve replacement in 1 patient $(1.5 \%)$.

\section{Survival and In-Hospital Complications}

Operative mortality was $2.5 \%(2 / 79)$. The first patient was a 29 -year-old African-American man who had aortic

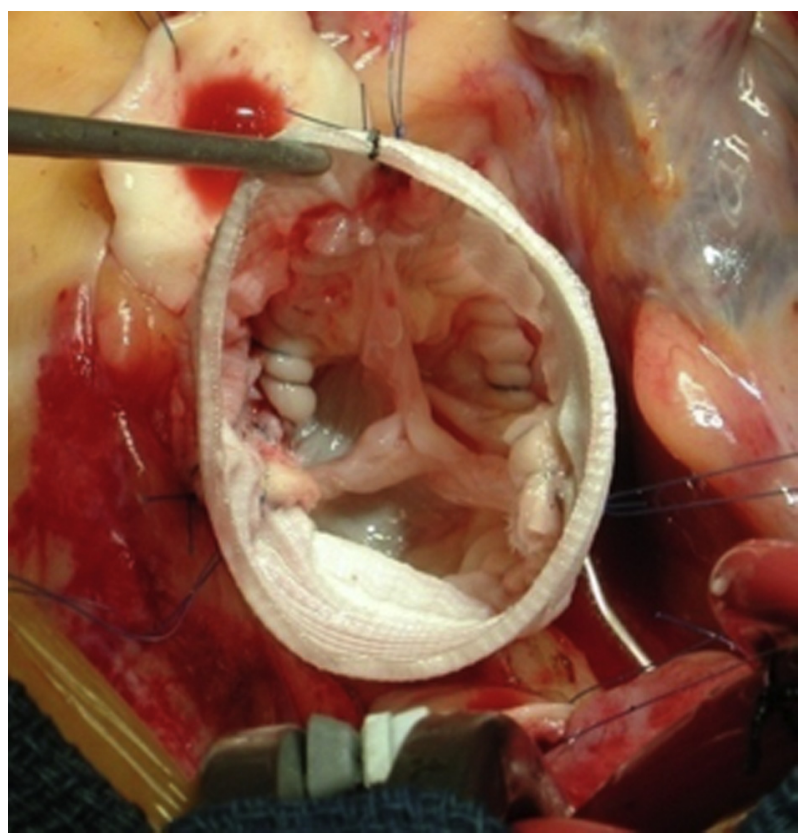

FIGURE 1. Reimplantation valve-sparing root replacement. 
root replacement and thoracoabdominal aortic aneurysm repair at another institution who presented to our institution in hemodynamic shock with massive hemoperitoneum. He required salvage exploratory laparotomy and right nephrectomy for renal artery rupture but did not survive. The second was a 16-year-old white female patient who had valvesparing root replacement at another institution and presented to us with an enlarging ascending aortic aneurysm. She underwent redo sternotomy and ascending aorta replacement but suffered a cardiac arrest in the immediate postoperative period requiring extracorporeal membrane oxygenation. She unfortunately developed disseminated intravascular coagulation, a large stroke, and passed away.

Late death occurred in 8 patients (8/77). Kaplan-Meier survival was $88 \%$ at 10 years $(n=9$ at risk at 10 years; Figure 2). Causes of death included subclavian dissection (intraoperative death during attempted repair), colon cancer, stroke, and unknown in 5 patients. In-hospital complications included bleeding requiring transfusion in 5, bleeding requiring reoperation in 1 , pneumonia in 3 , atrial fibrillation in 6 , and pneumothorax in 3 patients.

Nineteen patients (19/77) required subsequent operation (Table 1). Six had descending aorta replacement, 5 had ascending aorta replacement, 8 had aortic root replacement, and 10 had aortic arch replacement. Two patients required subsequent mitral valve repair/replacement, and 4 required repair of a coronary button aneurysm/pseudoaneurysm. Of the 61 patients who had isolated aortic root replacement, 4 required subsequent ascending repair and 7 required subsequent arch repair, with a mean time to reintervention of approximately 5 years. Of the 19 patients who required subsequent operations, 14 were alive at follow-up. Of the 5 who died, 4 were late deaths and 1 was an in-hospital mortality secondary to stroke after redo sternotomy and homograft aortic root and ascending replacement. Freedom-from subsequent operation was $47.8 \%$ at 10 years (Figure 3 ).

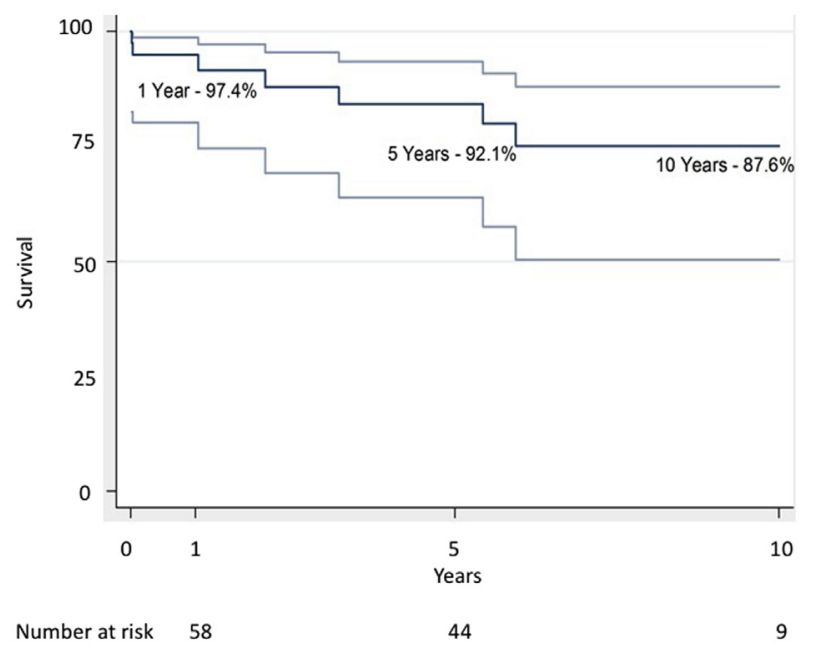

FIGURE 2. Kaplan-Meier survival curve.

\section{DISCUSSION}

Patients with LDS were stratified initially into 2 types depending on the severity of craniofacial features (type 1) or cutaneous features (type 2). ${ }^{3}$ The nosology was later revised because vascular disease is the most important concern in these patients. Four types of LDS are now described. ${ }^{4}$ The presence of an aneurysm or dissection along with a mutation in TGFBR1 (LDS 1), TGFBR2 (LDS 2), SMAD3 (LDS 3), or transforming growth factor beta 2 (LDS 4) is sufficient for the diagnosis of LDS (Table 2). Although variability in expression among the 4 LDS subtypes exists, medical and surgical management are similar.

Progressive aortic aneurysmal disease is a critical feature of LDS. Those with LDS 1 and 2 are at particularly greater risk for aortic catastrophe, and in our early experience these patients were found to rupture at early ages and at smaller aortic diameters than other connective tissue/aneurysmal syndromes. $^{1-3}$ Aortic dissection has been reported in children as young as 3 months of age. ${ }^{3,5}$ Early studies of LDS type 1 and 2 report aortic dissection and cerebral hemorrhage as the leading causes of death, with a mean age of death of 26 years. ${ }^{1-3}$ Early diagnosis, prompt treatment, and frequent surveillance should improve life expectancy. Table 3 lists our current guidelines for the cardiovascular care of patients with LDS.

Certain congenital heart defects are more common among patients with LDS compared with the normal population, including patent ductus arteriosus and mitral valve prolapse/insufficiency. These congenital defects should be addressed according to standard guidelines irrespective of the extent of aortic root disease. In our study, 3 patients had concomitant ligation of a patent ductus arteriosus at the time of index operation. Two patients underwent late mitral valve surgery, and 1 had concomitant mitral repair at the time of aortic root replacement.

The decision to proceed with aortic surgery is multifaceted and depends on aortic dimensions, rate of growth, aortic valve function, severity of noncardiac phenotype, family history, and aggressiveness of the known genotype. Although patients with Marfan syndrome show predilection for aortic dissection when the aortic root diameter reaches $5 \mathrm{~cm}$, patients with LDS are at risk for dissection when the aortic root reaches $4 \mathrm{~cm}$. Our current surgical guidelines are listed in Table 4. LDS type 1 and type 2 are clearly the more aggressive subtypes of LDS for which we recommend aortic root replacement at a threshold of $4 \mathrm{~cm}$. Within this subgroup, however, there are specific mutations known to be particularly aggressive and others associated with milder presentations. For these more aggressive genotypes, we consider surgery in childhood as soon as the annulus reaches $2 \mathrm{~cm}$ to accommodate a graft of adequate size to support growth. SMAD3 mutations found in LDS type 3 cause disease with intermediate severity. Aortic surgery is done at 4.0 to $4.5 \mathrm{~cm}$, again based on mutation and family history. 
TABLE 1. Reoperations

\begin{tabular}{lllr}
\hline Patient & \multicolumn{1}{c}{ Index operation } & \multicolumn{1}{c}{ Subsequent reoperation } & $\begin{array}{c}\text { Time to subsequent } \\
\text { reoperation, mo }\end{array}$ \\
\hline 1 & ARR & Redo ARR for acute AI and LCA pseudoaneurysm & 100 \\
2 & Arch repair & Arch pseudoaneurysm repair & 70 \\
3 & VSRR & Ascending and arch repair & 99 \\
4 & TAAA repair & ASD closure, AVr, and arch replacement & 61 \\
5 & ARR & ARR for aortic pseudoaneurysm & 28 \\
6 & VSRR & Ascending repair & 21 \\
7 & ARR, arch repair & Ascending graft infection requiring ARR, ascending, and arch & 12 \\
& & replacement with homograft & 72 \\
8 & ARR & Ruptured arch aneurysm repair & 106 \\
9 & VSRR & Arch aneurysm repair & 53 \\
10 & VSRR, AVr & Ascending/arch repair & 74 \\
11 & VSRR & AVR, aortic root enlargement, ascending and hemiarch repair & 153 \\
12 & ARR & RCA aneurysm plication & 98 \\
13 & VSRR & ARR, ascending, arch repair & 8 \\
14 & VSRR, CABG, arch repair & Aortic pseudoaneurysm repair \\
15 & FL sleeve & VSRR, AVr & 89 \\
16 & VSRR & Repair ruptured distal anastomotic aortic pseudoaneurysm & 29 \\
17 & FL sleeve & VSRR & 48 \\
18 & FL sleeve & VSRR & 7 \\
19 & ARR & RCA pseudoaneurysm & \\
\hline
\end{tabular}

$A R R$, Aortic root replacement; $A I$, aortic insufficiency; $L C A$, left coronary artery; VSRR, valve sparing root replacement; TAAA, thoracoabdominal aortic aneurysm; $A S D$, atrial septal defect; $A V r$, aortic valve repair; $A V R$, aortic valve replacement; $R C A$, right coronary artery; $C A B G$, coronary artery bypass grafting; $F L$, Florida.

LDS type 4 caused by mutations in transforming growth factor beta 2 is milder on the spectrum, and we would generally proceed with root replacement around $4.5 \mathrm{~cm}$, but may proceed with intervention earlier depending on the mutation and family history. The mean maximum aortic root diameter among the 65 patients with LDS who had aortic root replacement in this study was $4.17 \pm 0.97 \mathrm{~cm}$; the smallest root in this series was $2.0 \mathrm{~cm}$ and 21 of the 65 patients had root surgery with maximum root diameter $<4.0 \mathrm{~cm}$.

Valve-sparing root replacement has become a safe and reliable option for appropriately selected younger patients with LDS. ${ }^{6}$ Most of our patients have had successful valve-

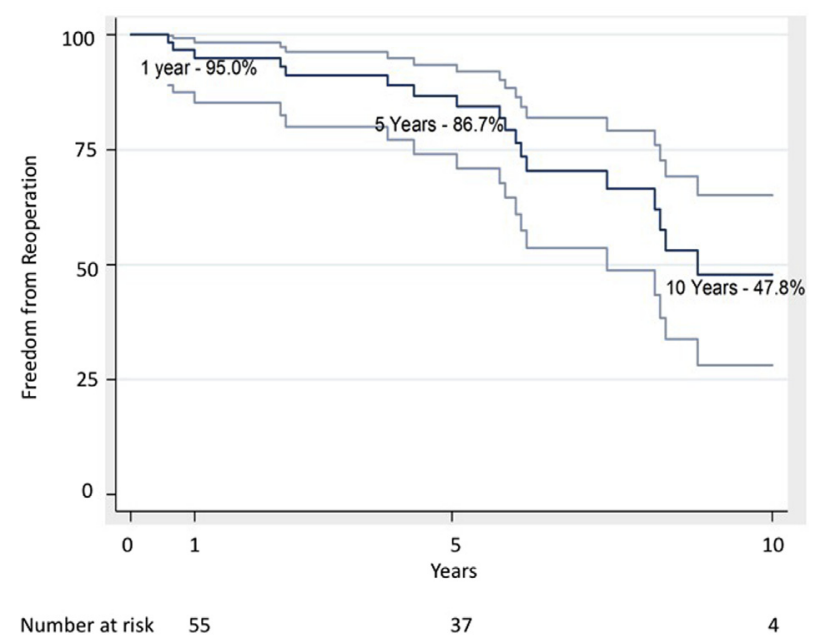

FIGURE 3. Freedom from reintervention. sparing surgery, with low risk of mortality and need for reoperation on the aortic root. Five patients who had valvesparing root replacement required reoperation on the aortic valve or root. Three of these 5 patients had a Florida sleeve procedure $^{7}: 1$ developed dilated coronary keyholes requiring redo valve-sparing root replacement, a second developed a "slipped" sleeve requiring redo valve-sparing root replacement, and a third developed annular dilation requiring redo valve-sparing root replacement and aortic valve repair.

Based on these initial outcomes with the Florida sleeve at our institution, we have abandoned this procedure in favor of conventional valve-sparing root replacement. One 9month-old child who had valve-sparing root replacement with a straight tube graft developed a dilated annulus, progressive aortic insufficiency, and a dilated arch and ascending aneurysm that required redo sternotomy, composite root replacement, and arch and ascending aneurysm repair 98 months after his initial operation. A second patient required composite root replacement 74 months after valvesparing root replacement after he developed severe aortic

TABLE 2. LDS classification

\begin{tabular}{lll}
\hline LDS type & Gene & \multicolumn{1}{c}{ Other disorders reported } \\
\hline 1 & $T G F B R 1$ & TAAD (previously, LDS 1a, 1b, 2a, 2b) \\
2 & $T G F B R 2$ & TAAD, MFS2 (previously, LDS 1a, 1b, 2a, 2b) \\
3 & $S M A D 3$ & Aneurysms-osteoarthritis syndrome \\
4 & $T G F B 2$ & $\begin{array}{c}\text { Aneurysm, aortic and cerebral, with arterial } \\
\text { tortuosity and skeletal manifestation }\end{array}$ \\
\hline
\end{tabular}

LDS, Loeys-Dietz syndrome; TGFBR, transforming growth factor-beta receptor $T A A D$, thoracic aortic aneurysm and dissection; MFS2, Marfan syndrome type 2; $S M A D 3$, mothers against decapentaplegic homolog 3 . 
TABLE 3. Guidelines for cardiovascular care

Routine cardiovascular care

1. Yearly echocardiogram, but may require shorter interval depending on extent of aortic disease

2. ARB, beta blocker, and/or ACEi for strict control of hypertension

3. Avoidance of contact/competitive sports, isometric exercises, strenuous exercise, blows to head/chest

4. Avoidance of stimulants and vasoconstrictors

5. SBE prophylaxis for those with artificial valves

6. Atrial fibrillation/arrhythmia management per normal protocol

7. Cardiac surgery consultation when approaching surgical thresholds for intervention

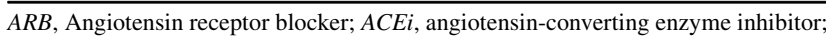
$S B E$, subacute bacterial endocarditis.

insufficiency secondary to the annulus prolapsing below the graft due to a torn subannular suture below the right coronary leaflet.

Our experience with valve-sparing root replacement has been described in detail elsewhere. ${ }^{6}$ Our operative technique for the majority of patients who had valve-sparing root replacement was a modified David V procedure in which the native aortic valve was resuspended within a Dacron graft with prefashioned pseudosinuses (Gelweave Valsalva graft; Vaskutek, Renfrewshire, Scotland, UK) (Video 1). Early in our experience, we adopted the Yacoub remodeling procedure because we felt that reconstructing the sinuses of Valsalva was important for long term aortic valve function; however, our reintervention rate was high due to annular dilatation and progressive aortic insufficiency. Subsequently, we switched to the reimplantation procedure when the Valsalva graft became available.

TABLE 4. Surgical thresholds for cardiovascular intervention in LDS 1,2 , and 3

\begin{tabular}{|c|c|}
\hline System & Threshold \\
\hline \multicolumn{2}{|l|}{ Aortic root } \\
\hline Children & $\begin{array}{l}\text { 1. Delay surgery until annulus }>2.0 \mathrm{~cm} \text { to } \\
\text { accommodate adult size graft } \\
\text { 2. Aortic root diameter of } 4 \mathrm{~cm} \\
\text { 3. Rapidly expanding aorta }(>0.5 \mathrm{~cm} / \mathrm{y}) \text {, severe } \\
\text { craniofacial features, strong family history of } \\
\text { early aortic catastrophe may warrant earlier } \\
\text { surgical intervention }\end{array}$ \\
\hline Adult & $\begin{array}{l}\text { Aortic root diameter }>4 \mathrm{~cm} \text { or rapidly expanding } \\
\quad>0.5 \mathrm{~cm} / \mathrm{y}\end{array}$ \\
\hline $\begin{array}{l}\text { Ascending } \\
\text { aorta/arch }\end{array}$ & $\begin{array}{l}\text { Aggressive surveillance if }>4 \mathrm{~cm} \text {, low threshold } \\
\text { if rapidly expanding }\end{array}$ \\
\hline $\begin{array}{l}\text { Descending } \\
\text { thoracic aorta }\end{array}$ & $4.5-5 \mathrm{~cm}$ or rapidly expanding $>1 \mathrm{~cm} / \mathrm{y}$ \\
\hline Abdominal aorta & $4-4.5 \mathrm{~cm}$ or rapidly expanding $>1 \mathrm{~cm} / \mathrm{y}$ \\
\hline $\begin{array}{l}\text { Visceral/iliac } \\
\text { arteries }\end{array}$ & $\begin{array}{l}\text { Individualized approach. Diameters } 2-3 \times \\
\text { expending diameter, or rapid expansion }\end{array}$ \\
\hline $\begin{array}{l}\text { Cerebrovascular } \\
\text { arteries }\end{array}$ & Individualized approach \\
\hline
\end{tabular}

Our contraindications to valve-sparing root replacement include the presence of severe leaflet fenestrations and leaflet asymmetry, acute dissection in the unstable patient, and bicuspid aortic valves with extensive calcification. For these patients, composite root replacement with a mechanical prosthesis remains a safe and reproducible option. Our valve-sparing root replacement operative technique, in general, has not changed in the LDS patient population. In a small number of children with LDS 1 and 2, we have placed additional subannular sutures in the area of the aorto-mitral curtain to improve stabilization, but our approach to the hemostatic suture line, distal anastomosis, and coronary reimplantation is the same.

The decision to perform aortic arch replacement at the time of aortic root replacement remains challenging. Many of our patients developed aneurysmal disease distal to the aortic root graft and in the aortic arch, which is likely secondary to disease progression and not the aortic root

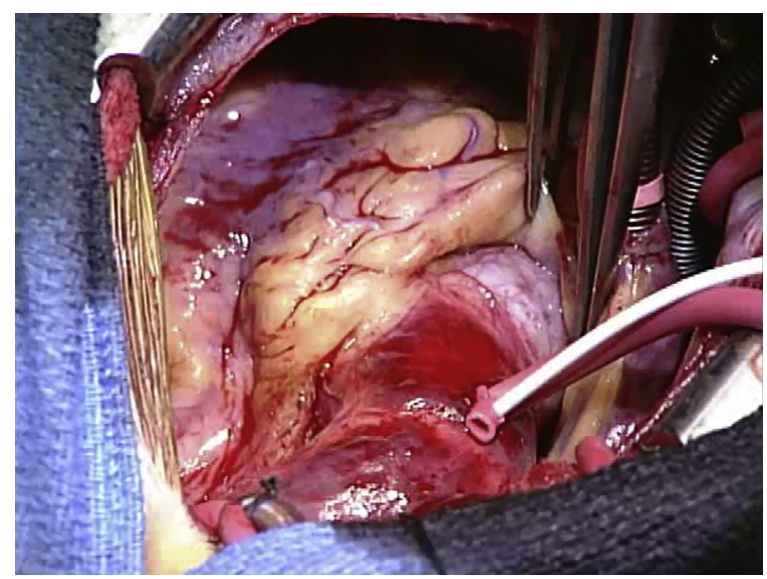

VIDEO 1. Valve-sparing aortic root replacement-surgical technique. Please see Cameron DE. Valve-sparing aortic root replacement. Semin Thorac Cardiovasc Surg Pediatr Card Surg Ann. 2012;15:20-3. In brief, the operation is performed via median sternotomy using central aortic and bicaval venous cannulation, venting via the right superior pulmonary vein, moderate systemic hypothermia to $28^{\circ} \mathrm{C}$, and blood cardioplegia. The right atrium is explored and a patent foramen ovale, if present, is closed in each case. The aorta is divided above the sinotubular junction and a graft that is 3 to $4 \mathrm{~mm}$ larger than the optimal sinotubular diameter that maximized leaflet apposition is chosen. The graft is trimmed, leaving 2 to 3 rings below the sinus segment and 4 to 5 rings above. Three pledgetted subannular mattress sutures are placed from within the left ventricular outflow tract outward and are used to secure the graft and ensure that the valve sits within the sinus of the graft. The commissures are fixed to the sinotubular junction of the graft with pledgetted mattress sutures. The annulus and sinus remnant are sewn to the inside of the graft with 4-0 or 5-0 polypropylene sutures. The coronary buttons are anastomosed to the sinus segments of the graft with external felt strip reinforcement. The valve is then inspected for prolapse, which can be addressed with leaflet plication or free edge suspension. Finally, the distal anastomosis is then performed with felt strip reinforcement. Video available at: http://www.jtcvsonline.org/article/ S0022-5223(16)31496-9/addons. 
operation itself. In the absence of aortic dissection, patients with LDS are more likely to require arch intervention after elective root replacement than their Marfan syndrome counterparts. In the presence of aortic dissection, we have found that the rate of arch interventions is similar between LDS and patients with Marfan syndrome. Our current practice is not to proceed with routine arch replacement in an effort to minimize aortic crossclamp time as well as to avoid additional suture lines that may increase the risk of bleeding. Given the increased rate of subsequent arch intervention, however, we believe it is justified to proceed with a short circulatory arrest time and arch replacement in selected patients. Currently, we adopt an individualized approach based on dimensions, rate of growth, severity of craniofacial features, genotype (particular mutations), and family history. To date, 1 patient who had composite aortic root replacement required late arch repair, 4 patients who had valve-sparing root replacement had late arch repair, 1 patient who had valve-sparing root replacement had subsequent hemi-arch repair, and 1 patient who had thoracoabdominal aneurysm repair had late arch repair.

Although others have used stent grafts successfully, ${ }^{8}$ we do not use thoracic stent grafts in patients with LDS routinely and prefer an open repair because stent grafts may result in late failure secondary to dilation of the fixation zone or ongoing perfusion through the false lumen. Furthermore, many patients with LDS have prohibitive vascular tortuosity that limits the use of endovascular delivery systems. In contrast, stent grafts may serve as a bridge postaortic dissection until the patient can be transferred to an experienced institution at which traditional open repair can be performed.

Postoperative surveillance of patients with LDS includes echocardiography every 3 to 6 months for the first year after surgery, which is then extended to every 6 months to 1 year. Full cardiovascular imaging should be obtained preoperatively with either magnetic resonance angiography or computed tomography angiography with 3-dimensional reconstruction, and then repeated after 1 year. The frequency of full-body imaging will be determined based on findings but should occur at least every 2 years. In particular, patients with type B dissections should be monitored aggressively for aneurysm growth. Currently, we recommend follow-up imaging at 7 to 14 days postdissection, then repeat imaging at $1,3,6$, and 12 months, and then yearly thereafter. As we discovered in our initial report, ${ }^{2} 24.6 \%$ (19/77) of our patients with LDS required repeat surgical interventions. This important finding emphasizes the need for strict and aggressive surveillance of the entire arterial tree.

In addition to aggressive surveillance and timely surgical intervention, optimal medical management is important. We routinely use beta blockade ${ }^{9}$ to reduce hemodynamic stress on the vasculature. Although others promote the use of angiotensin-converting enzyme inhibitors, ${ }^{10,11}$ we prescribe angiotensin receptor blockers because of their specific effects on the transforming growth factor beta signaling cascade. ${ }^{12}$

Our intermediate-term experience with LDS builds on our initial report from 2007. We have continued our aggressive approach with aortic root replacement and use a valvesparing strategy whenever possible. Although one-quarter of our patients required subsequent reintervention, we currently use an individualized approach to concomitant arch replacement based on dimensions, rate of growth, severity of craniofacial features/phenotype, genotype, and family history of early catastrophe. As subtypes of LDS are further described, meticulous and frequent cardiovascular imaging of the entire arterial tree remains essential for long-term management.

\section{Webcast}

You can watch a Webcast of this AATS meeting presentation by going to: http://webcast.aats.org/2016/Video/ Tuesday/05-17-16_Ballroom_I_1607_Patel-800.mp4.

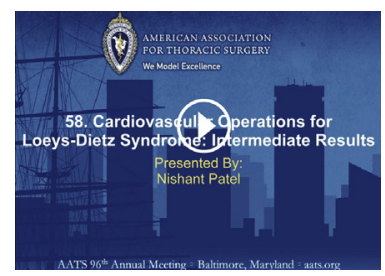

\section{Conflict of Interest Statement}

Authors have nothing to disclose with regard to commercial support.

\section{References}

1. Loeys BL, Chen J, Neptune ER, Judge DP, Podowski M, Holm T, et al. A syndrome of altered cardiovazscular, craniofacial, neurocognitive, and skeletal development caused by mutations in TGFBR1 or TGFBR2. Nat Genet. 2005;37:275-81.

2. Williams JL, Loeys BL, Nwakanma LU, Dietz HC, Spevak PJ, Patel ND, et al Early surgical experience with Loeys-Dietz: a new syndrome of aggressive thoracic aortic aneurysm disease. Ann Thorac Surg. 2007;83:S785-90.

3. Loeys BL, Schwarze U, Holm T, Callewaert BL, Thomas GH, Pannu H, et al. Aneurysm syndromes caused by mutations in the TGF-beta receptor. $N$ Engl J Med. 2006;355:788-98

4. MacCarrick G, Black JH III, Bowdin S, El-Hamamsy I, FrischmeyerGuerrerio PA, Guerrerio AL, et al. Loeys-Dietz syndrome: a primer for diagnosis and management. Genet Med. 2014;16:576-87.

5. Malhotra A, Westesson PL. Loeys-Dietz syndrome. Pediatr Radiol. 2009;39: 1015 .

6. Patel ND, Arnaoutakis GJ, George TJ, Allen JG, Alejo DE, Dietz HC, et al. Valve-sparing aortic root replacement in Loeys-Dietz syndrome. Ann Thorac Surg. 2011;92:556-60.

7. Hess PJ, Harman K, Klodell CT, Beaver TM, Bologna MT, Mikhail P, et al. Early outcomes using the Florida sleeve repair for correction of aortic insufficiency due to root aneurysms. Ann Thorac Surg. 2009;87:1161-8.

8. Williams JB, McCann RL, Hughes GC. Total aortic replacement in Loeys-Dietz syndrome. J Card Surg. 2011;26:304-8.

9. Shores J, Berger KR, Murphy EA, Pyeritz RE. Progression of aortic dilation and the benefit of long-term beta-adrenergic blockade in Marfan's syndrome. N Engl J Med. 1994;330:1335-41.

10. Yetman AT, Beroukhim RS, Ivy DD, Manchester D. Importance of the clinical recognition of Loeys-Dietz syndrome in the neonatal period. Pediatrics. 2007; 119:e1199-202. 
11. Everitt MD, Pinto N, Hawkins JA, Mitchell MB, Kouretas PC, Yetman AT. Cardiovascular surgery in children with Marfan syndrome or Loeys-Dietz syndrome. J Thorac Cardiovasc Surg. 2009;137:1327-32.

12. Matt P, Habashi J, Carrel T, Cameron DE, Van Eyk JE, Dietz HC. Recent advances in understanding Marfan syndrome: should we now treat surgical patients with losartan? J Thorac Cardiovasc Surg. 2008;135:389-94.

Key Words: aortic aneurysm, Loeys-Dietz syndrome

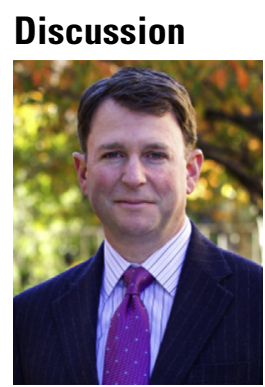

Dr M. Fischbein (Stanford, Calif). First, I would like to congratulate you on an excellent presentation. I think really the central message that you present today is that we can operate and do surgery on patients with LDS safely but that the reoperation rate is very high, and so we do have to follow these patients carefully.

I have 3 questions for you. One, you report that 5 patients required a redo operation for a redo root replacement after a valve-sparing procedure, 2 of these had the valve-sparing as opposed to the Florida sleeve, and this was due to annular dilatation. We have had a lot of discussions between Hopkins and Stanford about how many sutures you should place subannularly. At Stanford, we typically do about 15 and I know at Hopkins you do about 3. And so in patients with LDS, do you recommend or have you changed your technique? Do you do more subannular sutures knowing that there is a risk of recurrence from annular dilatation?

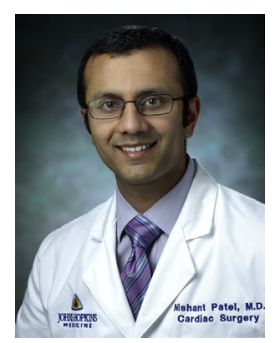

Dr Patel. Thank you, Dr. Fischbein, for that great question. We recognize your group's contribution to the management of this very challenging patient population. In terms of our valvesparing technique, we have not, in general, altered our approach. Our relative contraindications to valve-sparing surgery in this group are similar across diagnoses, namely, marked leaflet fenestrations, acute dissection in unstable patients, and severely calcified bicuspid aortic valves. In terms of technical nuances, in general, we have not changed our technique. There have been a couple of children with LDS type I and Type II that have required additional subannular sutures in the aortomitral curtain. Otherwise, we have stuck to our 3 subannular sutures. With regard to the hemostatic suture line, distal anastomosis and the coronary buttons, our technique is the same.

Dr Fischbein. For the second question, I think you mentioned at the end, in contrast to patients with Marfan syndrome, there is a much greater incidence in patients with LDS who require arch replacement after a valvesparing procedure. Can you comment on whether perhaps in aortic referral centers if we should be doing a total arch replacement during the initial index procedure? And perhaps maybe you can mention in those patients that you had to go back to do an aortic arch replacement as a second operation, was their arch of normal caliber during that initial index procedure?

Dr Patel. In the patients in whom we had to go back and perform arch interventions, the arch dimensions at the index operation were normal. I think this is one of the challenges with this particular syndrome.

In the absence of aortic dissection, patients with LDS are much more likely to require arch intervention than Marfan patients. In the presence of dissection, the rate of reintervention on the arch is similar between Marfan and patients with LDS.

In general, in adults, especially those patients that have severe noncardiac phenotypes and mutations that are known to be more lethal, it is justified to proceed with a total arch replacement. We are reluctant to use this strategy in children who present for elective aortic root replacement and have normal arch dimensions.

Dr Fischbein. Then the last question is, although it is controversial, you did show how you describe the 4 LDS subtypes, and I was wondering if you could teach us which of the four subtypes are the more aggressive types, and are the indications for surgery the same between all four or perhaps if it is a more benign phenotype should you operate on those at a bigger dimension?

Dr Patel. Thank you for this great question. I think the Holy Grail of managing these patients and other connective tissue disorders would be to identify the genotype and phenotype of those patients that are particularly at risk for rupture and dissection.

LDS type I, due to mutations in the transforming growth factor (TGF)-beta receptor 1 gene, and LDS type II, due to mutations in the TGF-beta receptor 2 gene, are the 2 more lethal LDS subtypes. In these patients we generally proceed with surgery at a root dimension of $4 \mathrm{~cm}$. Even in those subgroups, however, there are mutations that are particularly bad actors, and in those patients we proceed with surgery at even younger ages or in infancy. We try to wait until the annulus is $2 \mathrm{~cm}$ to accommodate adult size grafts.

LDS type III is a result of mutations in the SMAD3 gene. In general, these are mutations that lead to a more intermediate type of phenotype. In these patients we proceed with surgery at around 4 to $4.5 \mathrm{~cm}$, and, again, depending on the particular mutation and family history of early aortic catastrophe, we may proceed with surgery sooner.

LDS type IV results from mutations in the TGF-beta ligand 2 gene. These are generally the milder forms of LDS. We will proceed with surgery at around $4.5 \mathrm{~cm}$.

I spoke to Hal Dietz recently and there is a fifth subtype due to mutations in the TGF-beta ligand 3 gene. These, like LDS type IV, are milder in severity and we proceed at about $4.5 \mathrm{~cm}$, again, taking into account family history and particular mutations.

Dr Fischbein. Thank you very much. 